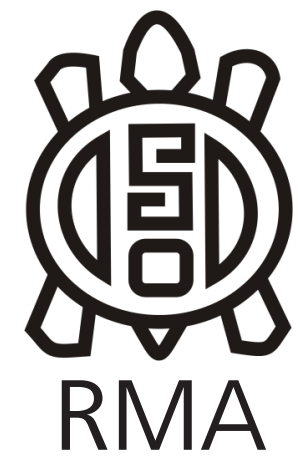

Dossier

\section{Avances y desafíos en torno a los abordajes cuali-cuatitativos aplicados a los estudios líticos}

\author{
Advances and challenges around the qualitative-quantitative \\ approaches applied to lithic studies
}

Marcelo Cardillo* y Federico Restifo**

*CONICET-IMHICIHU y UBA E-mail: marcelo.cardillo@gmail.com ${ }^{* *}$ CONICET-Instituto de Arqueología, Universidad de Buenos Aires, Argentina.

E-mail: federicorestifo@gmail.com
La larga tradición de los estudios de materiales líticos, así como su ubicuidad en el registro arqueológico a nivel espacial y temporal, han favorecido la gran diversificación de metodologías aplicadas a su identificación, registro y descripción. Asimismo, estos variados abordajes abarcan desde análisis puramente descriptivos y cualitativos a métodos cuantitativos que se encuentran anclados en gran medida a distintas epistemologías arqueológicas. Esta diversificación se ha intensificado recientemente en virtud del incremento de la capacidad de los sistemas informáticos de manejar crecientes bases de datos y de la proliferación de distintos software de análisis estadístico. Asimismo, la difusión de nuevos enfoques cuantitativos como la morfometría geométrica para el estudio de la forma o los análisis espaciales, abren nuevas posibilidades para el estudio de los conjuntos artefactuales. Por su parte, otro aspecto central es la construcción o elección de unidades de análisis como paso previo a la generación de datos. Éstas se encuentran en estrecha relación con el interrogante de investigación, lo que resulta clave a la hora de seleccionar variables y ordenar la variación observada. Sin embargo, no se ha dado un énfasis equivalente en la reflexión en torno a la forma en que estos distintos aspectos se articulan entre sí y de que manera responden de forma más o menos eficiente a los problemas planteados dentro de los estudios líticos.

Por lo tanto, en el marco del primer Congreso Argentino de Estudios Líticos, el simposio tuvo como objetivo presentar, comparar y discutir estos distintos abordajes en Argentina. Un ejemplo claro de esta diversidad de abordajes son los trabajos presentados en este dossier. Primeramente, los trabajos de Moreno \& Egea y por Montegú, los que se enfocan en una de las materias primas más complejas de ser estudiadas desde el punto de vista tecnológico y desde las estrategias de explotación: el cuarzo. Moreno \& Egea exploran la articulación de distintas variables como la talla experimental y la morfometría geométrica, con el fin de caracterizar las estrategias tecnológicas en los grupos humanos que habitaron Sierra de El Alto-Ancasti, provincia de Catamarca. Los autores identificaron algunas variables que fueron controladas mediante el proceso de reducción (como el espesor) así como en la confección de instrumentos (prefiriendo ángulos agudos). Por su parte la aplicación de morfometría geométrica, sugiere escasa formalidad en los diseños obtenidos. Por su parte, Montegú utiliza la técnica MANA para determinar las técnicas de explotación del cuarzo en El Alto, ubicado en el sector norte del cerro Ampuqcatao o Pelao (Valle de Tafí, Tucumán). El autor modela de esta manera, las distintas etapas de producción lítica del cuarzo en las distintas unidades del paisaje estudiadas. Los resultados le permitieron definir algunas etapas de reducción, incluyendo el modelo de aprovisionamiento más probable dentro de este contexto agropastoril.

Otro tema presente en los trabajos presentados en este dossier es la estandarización. El trabajo de Flores Coni y coautores, explora la variabilidad formal de los raspadores del centro-oeste de Santa Cruz, Patagonia meridional y su relación con la variabilidad espacio-temporal empleando técnicas estadísticas. Los resultados obtenidos a partir del estudio de la variación métrica, sugiere estabilidad en las estrategias de manufactura de raspadores entre el Holoceno medio y tardío.

Por último, Federico Restifo presenta una discusión sobre los criterios a tener en cuenta para la selección o construcción de unidades de análisis en estudios líticos, en base un acaso de estudio en la Puna salteña. El trabajo destaca el impacto de la selección y construcción de unidades (por ejemplo, por extensión o intención) en el proceso de investigación, en particular en la selección de los datos y en el contraste de hipótesis. Una de las conclusiones principales de este trabajo es 
la posible complementariedad entre ambos enfoques y en las distintas escalas de análisis siempre que ello esté contemplado al inicio de la investigación.

Si bien se trata de cuatro trabajos, este dossier refleja una diversidad de enfoques en el análisis lítico. Consideramos que esto constituye un punto de partida hacia la exploración de diferentes métodos y técnicas de análisis para abordar la diversidad de problemáticas que tienen lugar dentro de los análisis líticos en Argentina. 